\title{
Percutaneous cholecystostomy in the management of acute cholecystitis -10 years of experience
}

\author{
Petr Dvorak ${ }^{1}$, Petr Hoffmann ${ }^{1}$, Ondrej Renc ${ }^{1}$, Tomas Dusek ${ }^{2}$, Stanislav Rejchrt ${ }^{3}$, Ondrej Slezak ${ }^{1}$, Pavel Vyroubal ${ }^{4}$ \\ ${ }^{1}$ Department of Radiology, University Hospital, Hradec Kralove, Czech Republic \\ ${ }^{2}$ Department of Surgery, University Hospital, Hradec Kralove, Czech Republic \\ ${ }^{3} 2^{\text {nd }}$ Department of Internal Medicine - Gastroenterology, University Hospital, Hradec Kralove, Czech Republic \\ ${ }^{4} 3^{\text {rd }}$ Department of Internal Medicine - Metabolic Care and Gerontology, University Hospital, Hradec Kralove, Czech Republic
}

Videosurgery Miniinv 2019; 14 (4): 516-525

DOI: https://doi.org/10.5114/wiitm.2019.84704

\begin{abstract}
Introduction: The preferred treatment for acute cholecystitis is cholecystectomy, but for patients with precluded general anesthesia due to critical illness or multiple medical comorbidities it is not suitable. Cholecystostomy could be a minimally invasive therapeutic alternative.

Aim: To retrospectively evaluate the indications, technical features, efficacy, complications, patients' development and relationships among monitored parameters of percutaneous computed tomography (CT)-guided cholecystostomies in cases of acute cholecystitis and find the role of this procedure in appropriate treatment selection.

Material and methods: Over the course of 10 years, 75 percutaneous cholecystostomy procedures in 69 patients were performed in cases with diagnosed acute cholecystitis, precluded general anesthesia and contraindicated cholecystectomy by an experienced surgeon and anesthesiologist. These interventions were done using only local anesthesia. The patients were men in 39 cases and women in 33 cases, aged 33 to 91 years.

Results: Technical success was achieved in all cases. The indications were sepsis in 34 (45.3\%) cases, bridging acute gallbladder inflammatory status in 15 (20\%) interventions, serious medical comorbidities in 8 (10.7\%) cases, disseminated malignancy and cardiac failure in 6 cases each (both 8\%) and neurological affections in 5 (6.5\%) cases. Cholecystostomy was frequently the final solution in acalculous cholecystitis (79.3\%). The 30-day mortality rate was determined at $10.7 \%$ and the overall complication rate was $21.3 \%$, but all of these complications were managed conservatively or using minimally invasive treatment.

Conclusions: Percutaneous CT-guided cholecystostomy is reserved for patients with a serious medical status for various reasons that preclude surgical treatment and general anesthesia. Simultaneously, technical success and efficacy are high and the complication rate is acceptable.
\end{abstract}

Key words: indications, acute cholecystitis, cholecystostomy, gallbladder drainage.

\section{Introduction}

Acute cholecystitis is one of the most common surgical emergencies in industrially advanced countries. In European countries it describes an acute inflammation of the gallbladder wall. For acute gallbladder content affection, the term empyema is used. According to the severity the disease is divided into three grades - I (mild), II (moderate) and III (severe) - by the Tokyo guidelines 2018 (TG 2018) [1-3]. The preferred and recommended treatment option is

\section{Address for correspondence}

Petr Hoffmann MD, PhD, Department of Radiology, University Hospital Hradec Kralove, 581 Sokolska St, 50005 Hradec Kralove,

Czech Republic, phone: +42 0495833607, e-mail: petr.hoffmann@fnhk.cz 
cholecystectomy, performed using a laparoscopic or open approach [4, 5]. In mild inflammatory affection with minimally expressed clinical, imaging and laboratory symptoms a conservative approach is suitable.

This therapeutic scheme often cannot be applied to elderly patients with many medical comorbidities, where urgent surgical cholecystectomy morbidity and mortality reach $41 \%$ and $19 \%[6,7]$ respectively. Also, critically ill patients with multiple organ dysfunction syndrome and precluded general anesthesia need minimally invasive and simultaneously effective treatment options.

Cholecystostomy (or gallbladder drainage or gallbladder tube placement) was historically a surgical procedure. Surgeons completely replaced this technique with the cholecystectomy. The cholecystostomy returned to clinical practice with interventional radiology and the accessibility of imaging methods [8]. For critically ill and polymorbid patients, it presents tolerable and efficient therapeutic possibility.

\section{Aim}

The aim of our study was to retrospectively evaluate the indications, technical features, efficacy, complications, patients' development and relationships among monitored parameters of percutaneous computed tomography (CT)-guided cholecystostomies in cases of diagnosed acute cholecystitis and help to find the appropriate role in the whole treatment spectrum.

\section{Material and methods}

Over the course of ten years, from June 2008 to June 2018, a group of 75 patients was retrospectively evaluated. In all cases acute cholecystitis was diagnosed using clinical, laboratory and imaging examinations. Clinically, a palpable pain in the right lower costal margin was present. Increased serum levels of C-reactive protein and white blood cell count were detected by laboratory methods. Ultrasound imaging was the most preferred modality.

Monitored parameters were age, gender, drain gauge, indication (i.e. the main reason for precluding the operation), presence of gallstones, drainage length, subsequent cholecystectomy, microbiological culture of gallbladder content and bacterial antibiotics sensitivity, drainage approach through or out of hepatic parenchyma, 30-day mortality rate, com- plications and their possible solutions. Indications were the most monitored parameter.

All the interventional procedures were performed under CT guidance. The device Siemens Somatom Definition AS Plus (Siemens, Forchheim, Germany) was used. All the patients were indicated by the multidisciplinary council (surgeon, interventional radiologist and anesthesiologist or intensive care physician). Fully informed consent was obtained in all cases by the drainage performing physician or surgeon with an explanation of the procedure principles, the resulting consequences, possible complications, and their potential solutions. This study was approved by the institutional ethics review committee. The procedures were performed using drainage catheters with a pigtail ending and locking system (Bard Navarre Instrument, Bard, Covington, Georgia, USA). In all cases, a one-step approach was used; the coaxial technique was never performed. Ultrasound bedside guidance was never used especially due to more precise $\mathrm{CT}$ gallbladder focusing, controlling the track and verifying the final drain position.

The proper procedure was planned according to a preprocedural imaging examination. The patients' position on the CT table was carefully selected; in all cases a supine position with or without the right side underlaid and with the elevated right upper extremity was suitable. The shortest and safest distance was determined. The drain track was also planned to avoid vessels and to penetrate healthy hepatic parenchyma before reaching the gallbladder. If the anatomical relationships did not allow the transhepatic approach, access for drainage through the abdominal cavity was selected.

The entry point was defined by placing a skin mark. The distance and drain track angle were measured on the basis of a local anesthesia needle short $\mathrm{CT}$ imaging. The skin was disinfected and covered with sterile drapes, exposing only the entry site. In accordance with the predetermined route, an $8 \mathrm{~F} / 10 \mathrm{~F} /$ $12 \mathrm{~F} / 14 \mathrm{~F}$ of $29 \mathrm{~cm}$ length drain with pigtail ending and locking system was inserted in the proper position into the gallbladder. The fluid content was placed into at least two test tubes for microbiological culture and bacterial antibiotics sensitivity.

All procedures were performed using only local anesthesia (Trimecaine, Zentiva, Prague, Czech Republic); conscious sedation or general anesthesia was never needed. Parameters of blood coagulation, international normalized ratio (INR; lower 
than 1.5) and activated partial thromboplastin time (aPTT; lower than 1.5) were noted before the drainage. After the intervention, one series of CT scans in the same ranges as in the preprocedural examination was performed to exclude the possibility of early complications, bleeding being the complication looked for first (Photo 1). Correct drainage position was also controlled using this series of CT scans. The point of drain insertion into the skin was cleaned with a surgical scrub. The drain was fixed with one skin suture, attached to the collecting bag and flushed with saline. The duration of the whole procedure including all preparations never exceeded 30 min.

The majority of cases were treated by the intensive care unit of the internal or surgical hospital department. Other patients were monitored by the standard surgery department. The clinical status and laboratory parameters of inflammation were controlled; 5 to 10 days after the intervention, cholecysto-cholangiography through the inserted drain was performed in non-complicated cases. The drain position and bile duct patency were noted; modification of the drainage site was also possible.

\section{Statistical analysis}

For the study, retrospective data collection was used. For the basic quantitative statistical evaluation, median and interval data were used. These parameters were correlated with indications, drain gauge, presence of gallstones, subsequent cholecystectomy, drainage approach, 30-day mortality rate, and complications using Fisher's exact test with contingency tables. Qualitative statistical data were descriptively evaluated and quantitative parameters were found. The statistical importance was established at the level $p=0.05$. The statistical software NCSS 11 (NCSS, LLC, Kaysville, Utah, USA) was used.

\section{Results}

A total of 75 interventional procedures with accessible results in the hospital information system were included in the study. Seventy-five cholecystostomies in 69 patients were performed. The patients were men in 39 (56.5\%) cases and women in $30(43.5 \%)$ cases, aged 33 to 91 years (median: 71 years of age). Technical success, i.e. verified drain placement into the gallbladder and fluid aspiration,

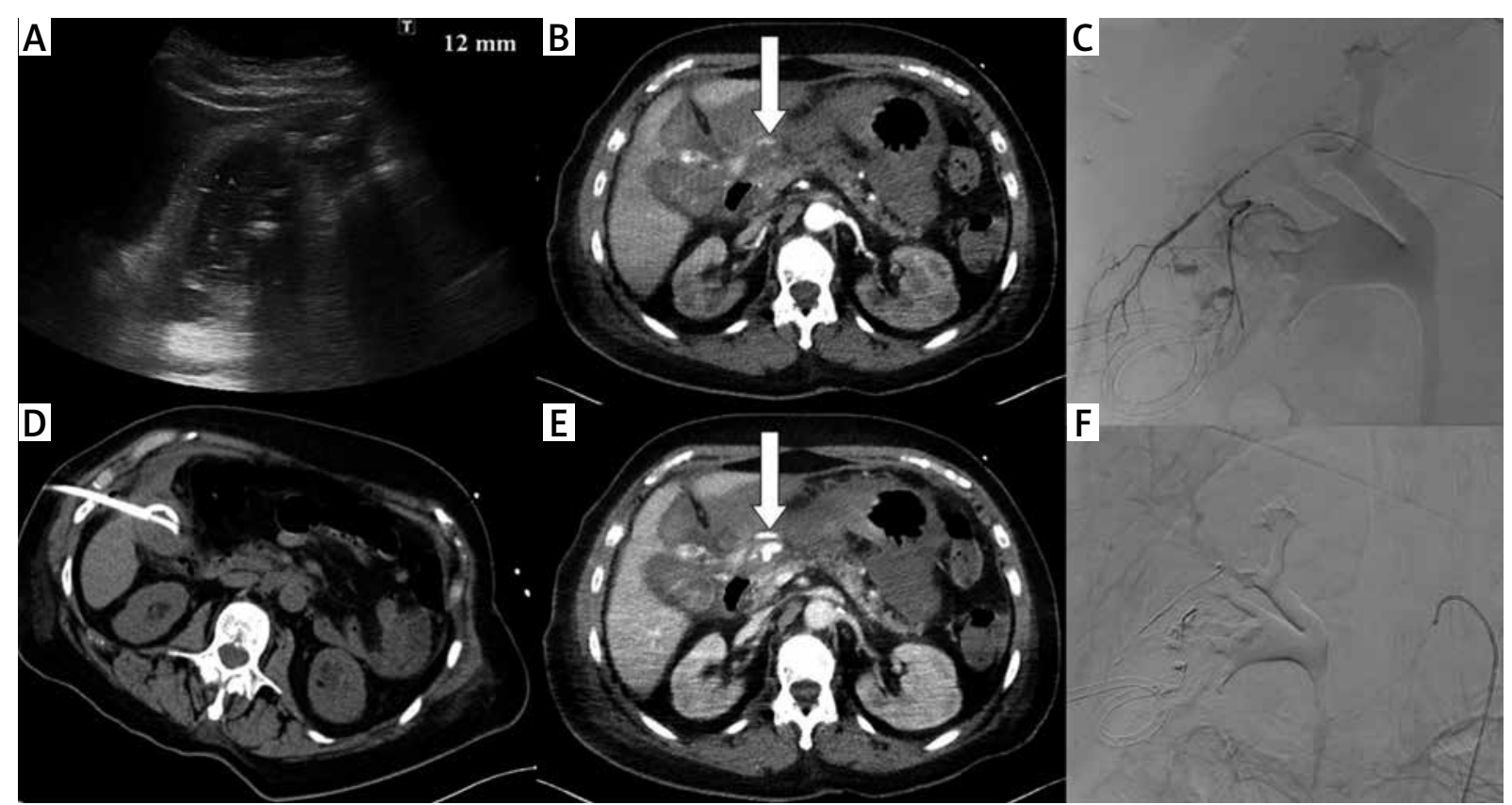

Photo 1. Complication. Acute calculous cholecystitis with infiltration of the gallbladder wall on ultrasound examination (A). Cholecystostomy was indicated due to pneumonia and performed using the transabdominal approach (B). 8 days after the procedure, bleeding into the drain and biliary ducts occurred. The contrast medium enhanced CT in arterial (C) and portal venous phase (D) revealed active hemorrhage (arrows) in the subhepatic space. Angiography was performed with active bleeding verification (E) and therapeutic embolisation with hemorrhage arrest (F) 
was achieved in all cases. The drainage length varied from 1 to 70 days (median length: 24.5 days).

In $6(8.7 \%)$ patients, two cholecystostomies were necessary. In all of these cases the first procedure was successful, and drains were extracted after clinical, laboratory and imaging normalization of the inflammatory gallbladder affection. Within the following 6 months, the situation re-occurred and the procedure was re-indicated.

The numbers of performed cholecystostomies and particular indications can be seen in Table I. In all bridging acute gallbladder inflammation cases gallstones were present, and also all these patients underwent cholecystectomy within the following two years. On the other hand only $20.6 \%$ of septic patients underwent surgical gallbladder treatment. Fisher's exact test proved a statistically significant relationship between the indications of septic patients and subsequent cholecystectomy ( $p=0.00002)$.

From a total of 75 procedures, $46(61.3 \%)$ were performed in calculous and 29 (38.7\%) in acalculous cholecystitis. Cholecystectomy was performed in 23 cases with the presence of gallstones, but in only 6 cases of acalculous inflammation. Consequent surgical treatment was significantly more frequent in patients with presence of gallstones ( $p=0.01492)$.

Within 30 days after the procedure, 8 patients died; the 30-day mortality rate was determined at $10.7 \%$. The specific causes were sepsis in 6 cases (lymphoma immunosuppression in 2 cases, and 1 case each of meningitis, necrotizing pancreatitis, fecal peritonitis and pneumonia related to chronic obstructive pulmonary disease), and in one case myocardial infarction and disseminated pancreatic malignancy.

The preferred way was planned through non-affected liver parenchyma before reaching the gallbladder. If the anatomical relationships did not allow this approach, then a route of drainage through the peritoneal space was used. The transhepatic approach was used in 41 (54.7\%) interventions and the transabdominal way in 34 (45.3\%) cases. Complications were revealed in the transabdominal drainage approach in 11 cases and using the transhepatic approach in 5 cases (Photo 2). Fisher's exact test proved a statistically significant relationship between the incidence of complications and the drainage approach ( $p=0.04778)$.

Complications were verified in a total of 16 cases, i.e. the overall complication rate was determined at 21.3\%. A detailed overview can be found in Table II.
Complications were revealed in 8 cases in septic patients, 4 times in bridging acute gallbladder inflammatory status, in 2 cases with disseminated malignancy, and one instance of medical comorbidity and cardiac failure. In 4 complicated cases there was acute cholecystitis complicated with surrounding perforation with several inflammatory fluid collections around the gallbladder and hepatic parenchyma. In two of these difficult cases cholecystostomy was successful (Photo 3). In 1 case cholecystostomy was performed in fulminant septic status due to emphysematous cholecystitis (Photo 4). The procedure was not successful and the patient had to be operated on due to vital indication and drainage dysfunction 1 day after the procedure.

Table I. The number of cholecystostomies and particular indications

\begin{tabular}{|c|c|c|}
\hline Cholecystostomy indications & $\begin{array}{c}\text { Number of } \\
\text { interventions }\end{array}$ & $\%$ \\
\hline Sepsis: & & 45.3 \\
\hline Necrotizing pancreatitis & 10 & \\
\hline Pneumonia and COPD & 8 & \\
\hline Lymphoma immunosuppression & 4 & \\
\hline Short bowel syndrome & 3 & \\
\hline Lower limb gangrene & 3 & \\
\hline Fecal peritonitis & 2 & \\
\hline Abdominal catastrophe & 2 & \\
\hline Duodenal ulcer perforation & 1 & \\
\hline Meningitis & 1 & \\
\hline Bridging acute inflammation & 15 & 20 \\
\hline Medical comorbidities & 8 & 10.7 \\
\hline Disseminated malignancy: & & 8 \\
\hline Pancreas cancer & 3 & \\
\hline Cholangiocarcinoma & 2 & \\
\hline Breast cancer & 1 & \\
\hline Cardiac failure: & & 8 \\
\hline Myocardial infarction & 5 & \\
\hline Pulmonary embolism & 1 & \\
\hline Neurological affections: & & 6.5 \\
\hline Stroke & 4 & \\
\hline Subarachnoid hemorrhage & 1 & \\
\hline Traumatic injury & 1 & 1.5 \\
\hline$N$ & 75 & \\
\hline
\end{tabular}




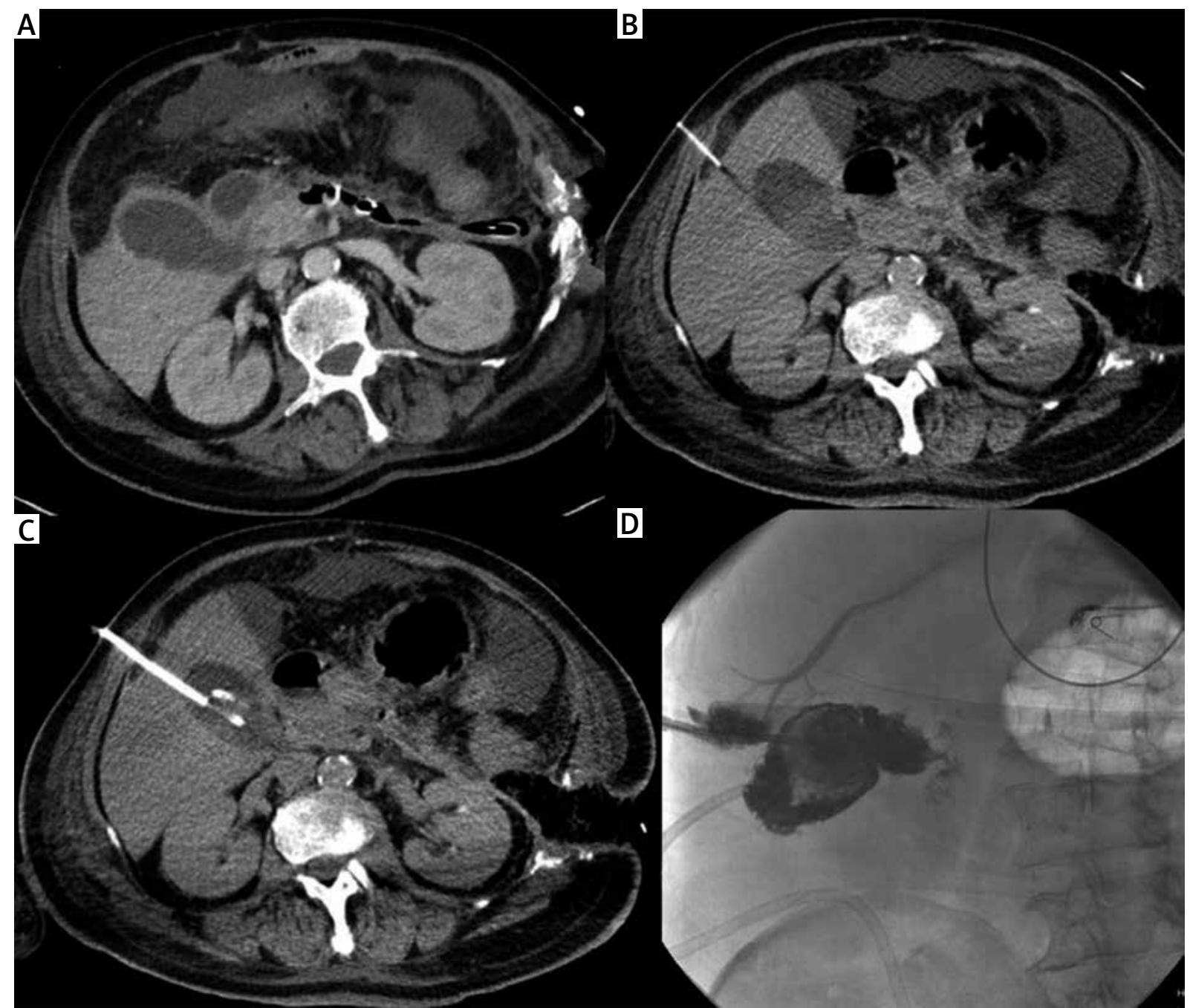

Photo 2. Incidental finding. Acute cholecystitis with enlargement of the gallbladder wall on the contrast medium enhanced CT examination (A). Cholecystostomy was indicated due to septic status related to necrotizing pancreatitis and performed using local anesthesia (B) and the transhepatic approach (C). Seven days after the intervention, routine cholecysto-cholangiography through the inserted drain was performed and revealed the communication with the hepatic vein (D)

Table II. The number of verified complications and their correlation with the inserted drain track

\begin{tabular}{|lccc|}
\hline Complications & Number & \multicolumn{2}{c|}{ Approach } \\
\cline { 2 - 4 } & & $\begin{array}{c}\text { Trans- } \\
\text { abdominal }\end{array}$ & Transhepatic \\
\hline Hemorrhage & 2 & 1 & 1 \\
\hline Drain extraction & 3 & 3 & 0 \\
\hline Drain dislocation & 4 & 2 & 2 \\
\hline Bile leakage & 3 & 2 & 1 \\
\hline Perforation & 4 & 3 & 1 \\
\hline$N$ & 16 & 11 & 5 \\
\hline
\end{tabular}

The $8 \mathrm{G}$ drain was used in 30 (40\%) cholecystostomies, the $10 \mathrm{G}$ in 21 (28\%) interventions, the $12 \mathrm{G}$ in $22(29.3 \%)$ cases and the $14 \mathrm{G}$ in 2 (2.7\%) cases. Fisher's exact test did not show a statistically significant relationship between the inserted drain gauge and the complication rate $(p=0.77596)$. A wider drain caliber, at least $12 \mathrm{G}$, can be used without increased incidence of complications; simultaneously the washing out of inflammatory gallbladder content remains sufficient.

A total of 15 different microbiological agents were proved in aspirated gallbladder content. An overview of verified microbiological agents is pre- 


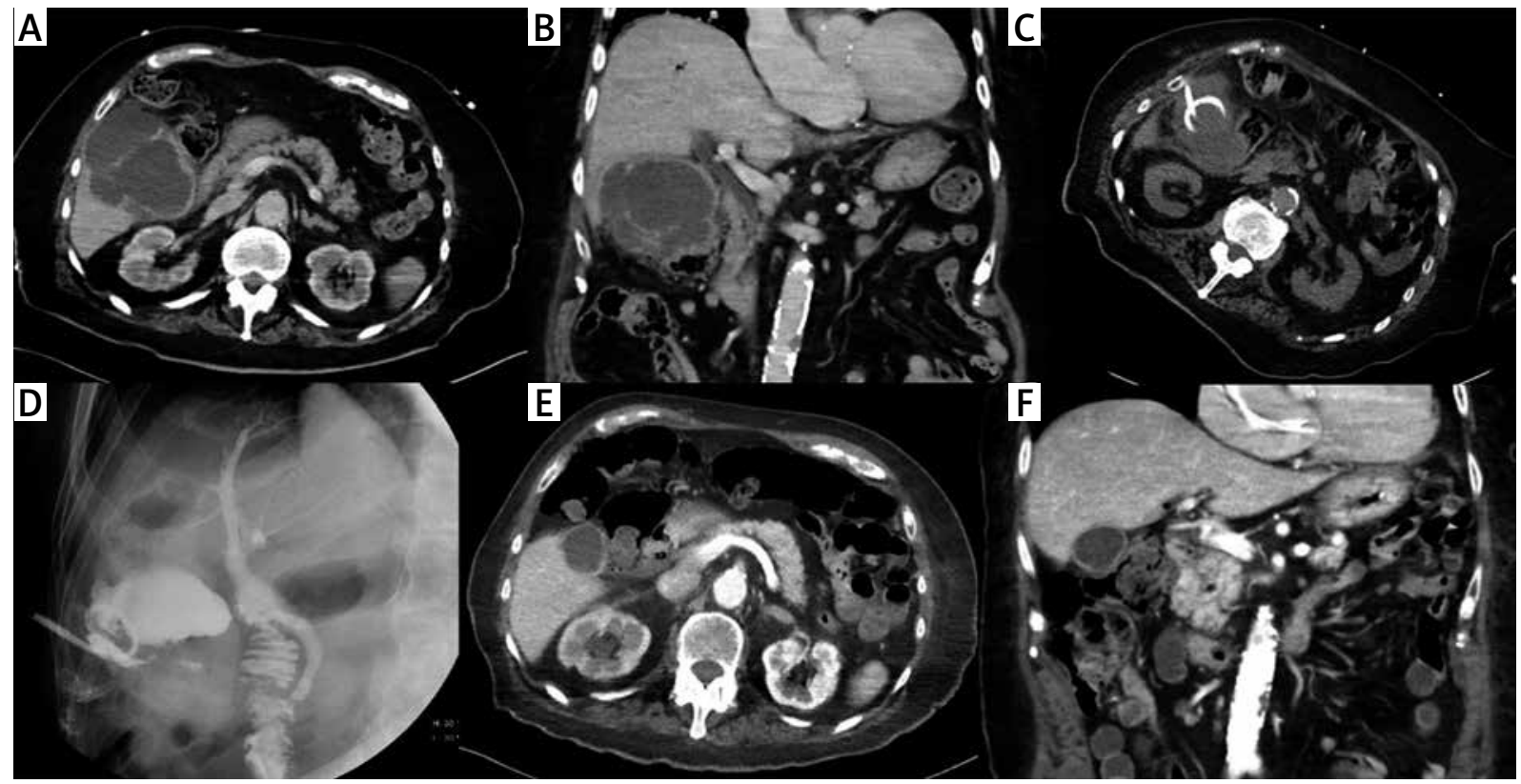

Photo 3. Cholecystostomy in case of acute cholecystitis complicated with surrounded perforation. Preprocedural contrast medium enhanced CT revealed several inflammatory fluid collections around the gallbladder in the transversal (A) and coronal plane (B). The drain was inserted using a transabdominal approach (C). Cholecysto-cholangiography through the drain was performed 7 days after the procedure and revealed no biliary peritonitis; the biliary tract was unobstructed (D). Contrast medium enhanced CT in transversal (E) and coronal plane (F) after 3 months confirmed an almost normal gallbladder pattern
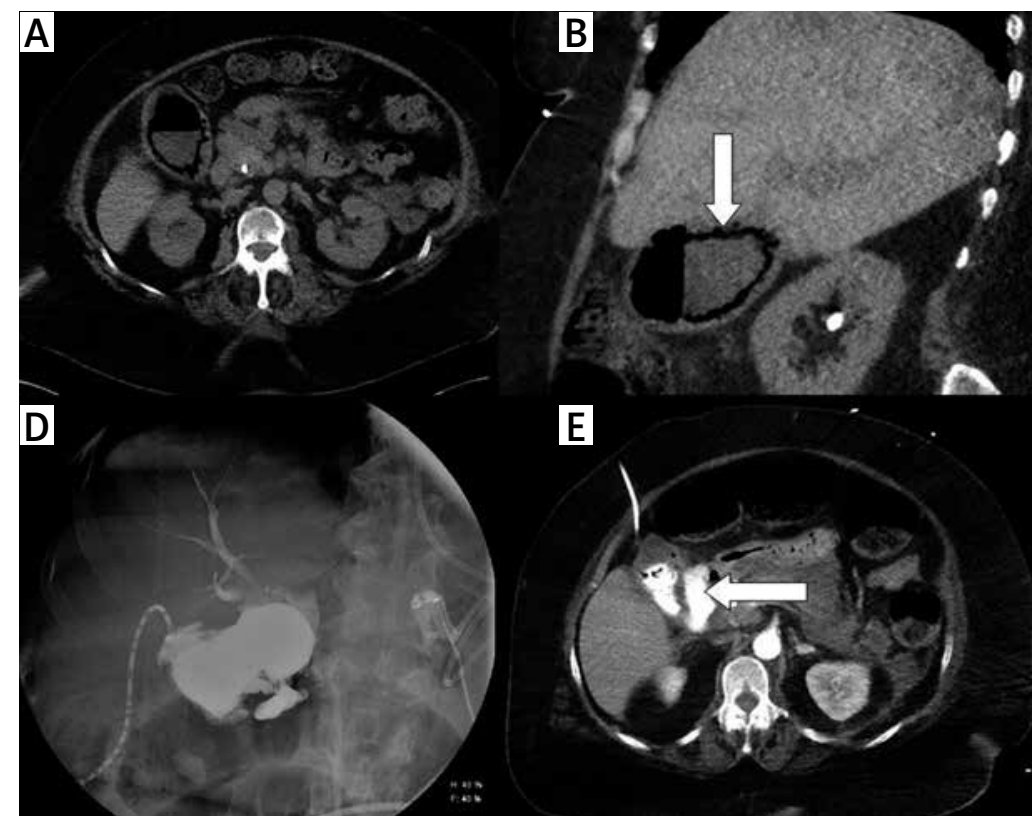

E

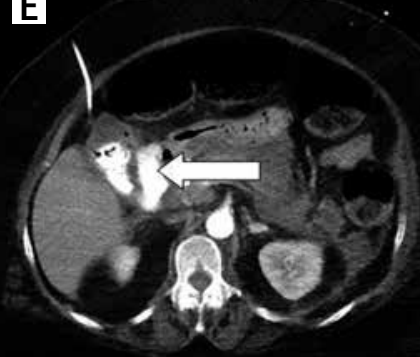

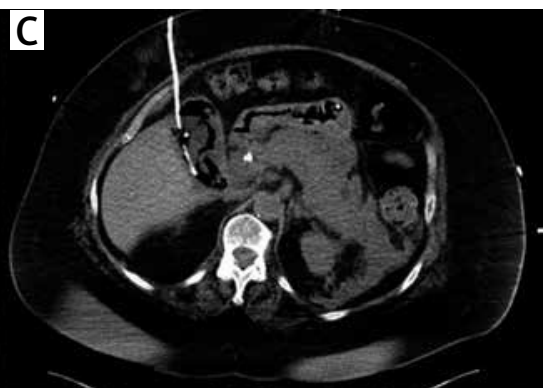

$\mathbf{F}$

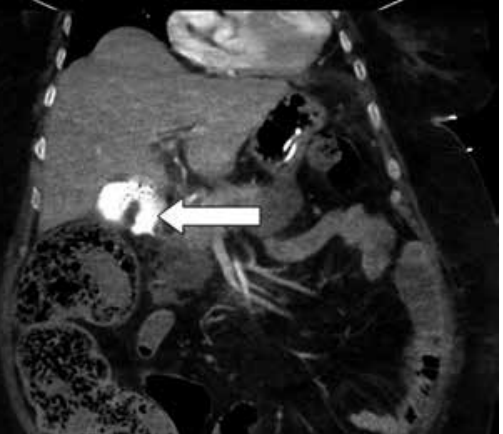

Photo 4. A case of fulminant septic status and cholecystostomy performed in emphysematous cholecystitis. Nonenhanced CT examination in transversal (A) and coronal plane (B) revealed a gallbladder wall containing gas (arrow). The drain was inserted using the transabdominal approach (C). Cholecysto-cholangiography through the drain was performed 7 days after the procedure and revealed discontinuity of the gallbladder wall (D). Computed tomography examination was performed immediately after cholangiography and confirmed the perforation in transversal $(\mathbf{E})$ and coronal plane $(\mathbf{F})$; leakage is noted with arrows 
Table III. The number of proved microbiological agents

\begin{tabular}{|lc|}
\hline Microbiological culture & Number \\
\hline Escherichia coli & 22 \\
\hline Enterococcus faecalis & 12 \\
\hline Pseudomonas aeruginosa & 8 \\
\hline Streptococcus anginosus & 6 \\
\hline Candida glabrata & 4 \\
\hline Staphylococcus aureus & 3 \\
\hline Candida albicans & 2 \\
\hline Klebsiella pneumoniae & 2 \\
\hline Enterobacter species & 1 \\
\hline Fusobacterium species & 1 \\
\hline Klebsiella oxytoca & 1 \\
\hline Lactobacillus gasseri & 1 \\
\hline Peptostreptococcus species & 1 \\
\hline Staphylococcus haemolyticus & 1 \\
\hline Streptococcus downei & 1 \\
\hline Negative & 9 \\
\hline N & 75 \\
\hline
\end{tabular}

sented in Table III. Negative cultivation results were revealed in $9(12 \%)$ cases. The patients with microbiologically negative cultivation from cholecystostomy drains had never undergone surgical gallbladder treatment $(p=0.0104)$.

\section{Discussion}

We report our data regarding the role of CT-guided percutaneous cholecystostomy in the management of acute cholecystitis. Technical success was achieved in all cases. The 30-day mortality rate was determined at $10.7 \%$. The overall complication rate was $21.3 \%$, but none of these complications were life-threatening, and were managed conservatively or using minimally invasive treatment. The most common indication was sepsis (45.3\%); only $20.6 \%$ of septic patients underwent surgical cholecystectomy in the patients' subsequent medical history. All cases of bridging acute gallbladder inflammation underwent surgical treatment within the next 2 years. Cholecystostomy was frequently the final solution in acalculous cholecystitis ( $79.3 \%$ of cases).
The transhepatic drainage approach was confirmed to be safer than the transabdominal way. Cholecystostomy was repeated in $8.7 \%$ of patients.

Gallbladder drainage is a final treatment significantly more frequently in cases of acute acalculous cholecystitis [9, 10]. Our experience corresponds with this fact. Cholecystitis with the presence of gallstones should be followed and treated surgically; an interval of 4-6 weeks between the drainage and cholecystectomy has been proven to have good outcomes for the following factors: volume of bleeding, operating time, percentage of patients switched to open surgery, and incidence of complications [7, 11]. The optimization of the time interval between cholecystostomy and subsequent cholecystectomy is indicated as a "future research question" by Tokyo guidelines 2018 (TG 2018) [12].

The definite advantages of percutaneous cholecystostomy are its low complication rate, rapidity of the procedure, efficiency and reproducibility [13, 14]. Its technical success is enormously high, almost $100 \%$, e.g. $98.9 \%$ [15]. The clinically successful effect reaches up to $85-92 \%[15,16]$. Therapeutic failure immediately followed by the surgery is reported in less than $5 \%$ [17] of cases. The 30-day mortality rate is reported to be between 0 and $25 \%$ [18-20]. The transhepatic drainage approach is preferred to the transabdominal way due to the lower complication rate [21]. Our experience corresponds to these published data. In the literature, articles are also available which revealed a non-significant difference in complication rates between the transhepatic and transperitoneal approaches [22, 23].

In non-surgical candidates, a conservative treatment is suitable at first; in cases of failure and inflammatory status worsening, biliary drainage is necessary $[24,25]$. Percutaneous gallbladder drainage is currently recommended before endoscopic retrograde cholangiopancreatography (ERCP) in grade II and III affection [3, 26]. In acalculous cholecystitis there is a high probability of a definitive solution; in calculous affection with gallbladder outlet obstruction the internal biliary drainage via ERCP, although technically excellent, cannot be successful and the suppurative content has to be washed out of the body using cholecystostomy (Photo 5) [27]. The recurrence rate is high in patients with calculous cholecystitis, up to $60 \%$ [28]. So, this is the reason for early interval prophylactic surgery once the patients' condition allows the safe use of general anesthesia. 


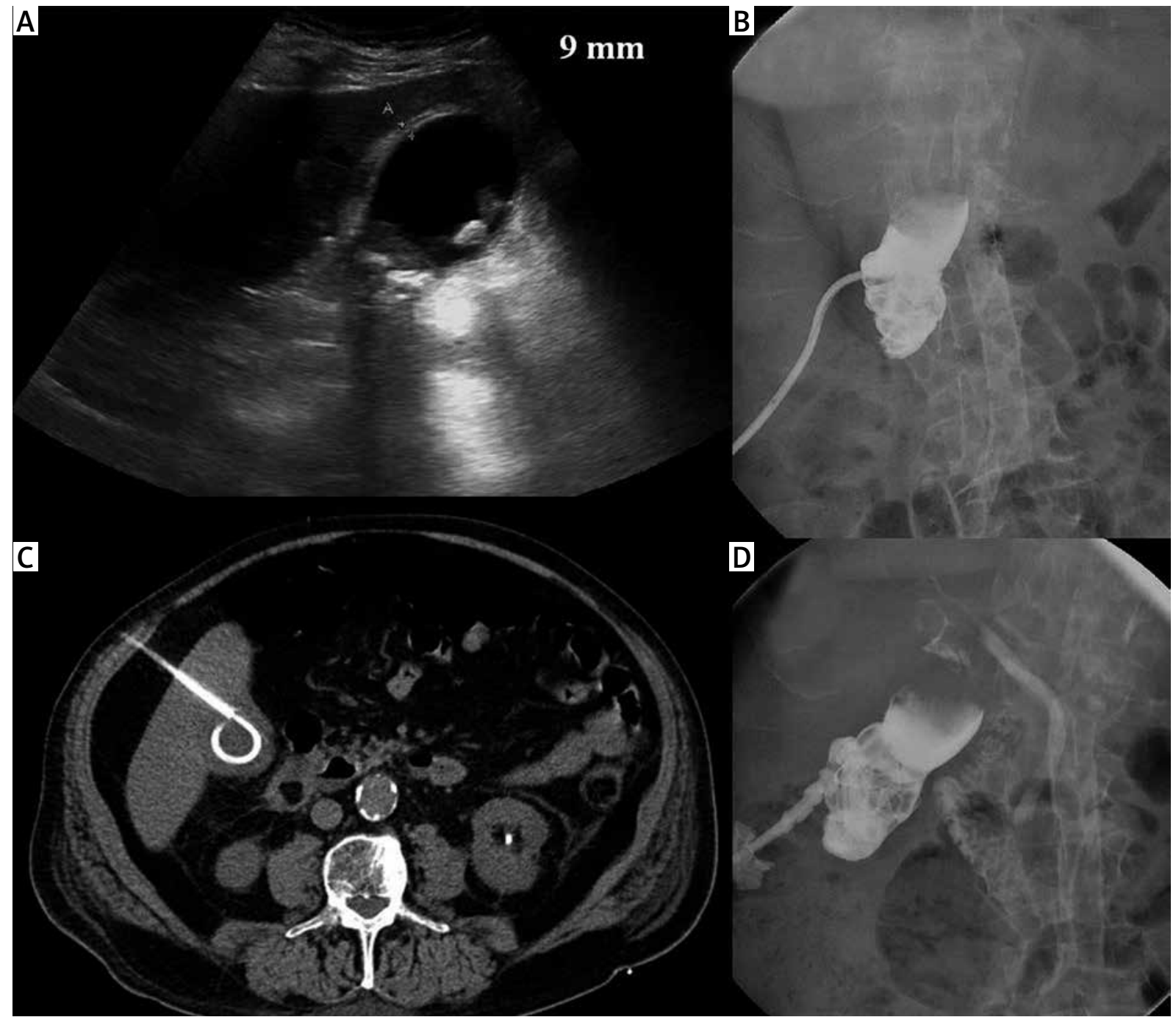

Photo 5. Cholecystostomy in blocked gallbladder. Acute calculous cholecystitis was diagnosed using ultrasound examination (A). Cholecystostomy using the transhepatic approach was performed (B). Cholecystocholangiography performed 8 days after the intervention revealed a completely obstructed gallbladder (C). In this period the internal biliary drainage cannot be effective; the suppurative content has to be washed out of the body via cholecystostomy. In the interval of the next 14 days the biliary ducts resumed normal patency into the duodenum (D)

The most common reported complications are drain dislodgment and bile leakage. The dislocations are published less frequently when using drains with a locking system. Cases of other complications bleeding, hemobiliary fistula, liver abscess, bowel injury or pneumothorax - are rarely reported [20]. The overall procedure-related complication rate varies in broad intervals; the majority of articles report the incidence under $20 \%$. Some studies report an overall complication rate of up to $69 \%$ [17]. Our experience corresponds to a $20 \%$ level.
The limits of our study are the retrospective data analysis, which depends on the hospital information system, absence of a control group, single centre data collection, smaller number of patients (but comparable with many published papers), and the individual indications depending on the actual specific patients' status evaluation and surgeons' clinical judgment.

\section{Conclusions}

Cholecystostomy using CT guidance and the percutaneous approach is a useful therapeutic alter- 
native to surgery in critically ill patients or in those with serious medical comorbidities that preclude general anesthesia. Technical and also clinical success is high, and the complication rate is acceptable. In calculous cholecystitis this procedure should be followed by cholecystectomy due to the high recurrence rate; in acalculous affection cholecystostomy is frequently a final solution. Despite favorable results, firm indication criteria are lacking and in specific clinical situations, surgeons' final decisions are critically important.

\section{Acknowledgments}

The authors are grateful to Dr. Olga Cermakova for considerable help with statistical data analysis, to Romana Brezinova for excellent technical support, and to Gwyneth Hamann and James Lago Chek MD for the language correction of the manuscript.

This work was supported by MH CZ - DRO (UHHK, 00179906), by MH CZ NT13531-3/2013 and by SVV 260398/2017.

\section{Conflict or interest}

The authors declare no conflict of interest.

\section{References}

1. Yokoe M, Hata J, Takada T, et al. Tokyo Guidelines 2018: diagnostic criteria and severity grading of acute cholecystitis (with videos). J Hepatobiliary Pancreat Sci 2018; 25: 41-54.

2. Okamoto K, Suzuki K, Takada T, et al. Tokyo Guidelines 2018 flowchart for the management of acute cholecystitis. J Hepatobiliary Pancreat Sci 2018; 25: 55-72.

3. Mori Y, Itoi T, Baron TH, et al. Tokyo Guidelines 2018: management strategies for gallbladder drainage in patients with acute cholecystitis (with videos). J Hepatobiliary Pancreat Sci 2018; 25: 87-95.

4. Wakabayashi G, Iwashita Y, Hibi T, et al. Tokyo Guidelines 2018 surgical management of acute cholecystitis: safe steps in laparoscopic cholecystectomy for acute cholecystitis (with videos). J Hepatobiliary Pancreat Sci 2018; 25: 73-86.

5. Bartosiak K, Liszka M, Drazba T, et al. Unexpected pathological findings after laparoscopic cholecystectomy - analysis of 1131 cases. Videosurgery Miniinv 2018; 13: 62-6.

6. Smith TJ, Manske JG, Mathiason MA, et al. Changing trends and outcomes in the use of percutaneous cholecystostomy tubes for acute cholecystitis. Ann Surg 2013; 257: 1112-5.

7. El-Gendi A, El-Shafei M, Emara D. Emergency versus delayed cholecystectomy after percutaneous transhepatic gallbladder drainage in grade II acute cholecystitis patients. J Gastrointest Surg 2017; 21: 284-93.

8. Radder RW. Ultrasonically guided percutaneous catheter drainage for gallbladder empyema. Diagn Imaging 1980; 49: 330-3.
9. Kirkegård J, Horn T, Christensen SD, et al. Percutaneous cholecystostomy is an effective definitive treatment option for acute acalculous cholecystitis. Scand I Surg 2015; 104: 238-43.

10. Noh SY, Gwon DI, Ko GY, et al. Role of percutaneous cholecystostomy for acute acalculous cholecystitis: clinical outcomes of 271 patients. Eur Radiol 2018; 28: 1449-55.

11. Karakayali FY, Akdur A, Kirnap M, et al. Emergency cholecystectomy vs percutaneous cholecystostomy plus delayed cholecystectomy for patients with acute cholecystitis. Hepatobiliary Pancreat Dis Int 2014; 13: 316-22.

12. Macchini D, Degrate L, Oldani M, et al. Timing of percutaneous cholecystostomy tube removal: systematic review. Minerva Chir 2016; 71: 415-26.

13. Gulaya K, Desai SS, Sato K. Percutaneous cholecystostomy: evidence-based current clinical practice. Semin Intervent Radiol 2016; 33: 291-6.

14. Papis D, Khalifa E, Bhogal R, et al. Is percutaneous cholecystostomy a good alternative treatment for acute cholecystitis in high-risk patients? Am Surg 2017; 83: 623-7.

15. Winbladh A, Gullstrand P, Svanvik J, Sandström P. Systematic review of cholecystostomy as a treatment option in acute cholecystitis. HPB (Oxford) 2009; 11: 183-93.

16. Ozyer U. Long-term results of percutaneous cholecystostomy for definitive treatment of acute acalculous cholecystitis: a 10year single-center experience. Acta Gastroenterol Belg 2018; 81: 393-7.

17. Friedrich AU, Baratta KP, Lewis J, et al. Cholecystostomy treatment in an ICU population: complications and risks. Surg Laparosc Endosc Percutan Tech 2016; 26: 410-6.

18. Sanjay P, Mittapalli D, Marioud A, et al. Clinical outcomes of a percutaneous cholecystostomy for acute cholecystitis: a multicentre analysis. HPB (Oxford) 2013; 15: 511-6.

19. Atar E, Bachar GN, Berlin S, et al. Percutaneous cholecystostomy in critically ill patients with acute cholecystitis: complications and late outcome. Clin Radiol 2014; 69: 247-52.

20. McKay A, Abulfaraj M, Lipschitz J. Short- and long-term outcomes following percutaneous cholecystostomy for acute cholecystitis in high-risk patients. Surg Endosc 2012; 26: 1343-51.

21. Little MW, Briggs JH, Tapping CR, et al. Percutaneous cholecystostomy: the radiologist's role in treating acute cholecystitis. Clin Radiol 2013; 68: 654-60.

22. Duncan C, Hunt SJ, Gade T, et al. Outcomes of percutaneous cholecystostomy in the presence of ascites. J Vasc Interv Radiol 2016; 27: 562-6.

23. Beland MD, Patel L, Ahn SH, Grand DJ. Image-guided cholecystostomy tube placement: short- and long-term outcomes of transhepatic versus transperitoneal placement. AJR Am J Roentgenol 2019; 212: 201-4.

24. Hall BR, Armijo PR, Krause C, et al. Emergent cholecystectomy is superior to percutaneous cholecystostomy tube placement in critically ill patients with emergent calculous cholecystitis. Am J Surg 2018; 216: 116-9.

25. Ahmed O, Rogers AC, Bolger JC, et al. Meta-analysis of outcomes of endoscopic ultrasound-guided gallbladder drainage versus percutaneous cholecystostomy for the management of acute cholecystitis. Surg Endosc 2018; 32: 1627-35. 
26. Simorov A, Ranade A, Parcells J, et al. Emergent cholecystostomy is superior to open cholecystectomy in extremely ill patients with acalculous cholecystitis: a large multicenter outcome study. Am J Surg 2013; 206: 935-40.

27. Li YL, Wong KH, Chiu KW, et al. Percutaneous cholecystostomy for high-risk patients with acute cholangitis. Medicine (Baltimore) 2018; 97: e0735.

28. Morse BC, Smith JB, Lawdahl RB, Roettger RH. Management of acute cholecystitis in critically ill patients: contemporary role for cholecystostomy and subsequent cholecystectomy. Am Surg 2010; 76: 708-12.

Received: 9.01.2019, accepted: 16.03.2019. 